\title{
A CHARACTERIZATION OF MINIMAL HAUSDORFF SPACES
}

\author{
LARRY L. HERRINGTON AND PAUL E. LONG
}

AbSTRACT. This paper gives a characterization of minimal Hausdorff spaces.

1. Preliminary definitions and theorems. A net $\theta: \mathscr{D} \rightarrow X r$-converges ${ }^{1}$ to $x_{0} \in X$ if for each open $V$ containing $x_{0}$, there exists a $d \in \mathcal{D}$ such that $\vartheta\left(T_{d}\right) \subset \mathrm{cl}(V)$ [2]. A net $\Theta: \mathcal{D} \rightarrow X r$-accumulates to $x_{0} \in X$ if for each open $V \subset X$ containing $x_{0}$ and for every $d \in \mathscr{D}, \mathcal{O}\left(T_{d}\right) \cap \mathrm{cl}(V) \neq \varnothing$. Theorem 5 of [1] shows that a Hausdorff space $X$ is minimal Hausdorff if and only if each net in $X$ with a unique $r$-accumulation point is convergent.

A function $f: X \rightarrow Y$ has a strongly-closed graph if for each $(x, y)$ $\in G(f)(G(f)$ denotes the graph of $f)$ there exist open sets $U \subset X$ and $V$ $\subset Y$ containing $x$ and $y$, respectively, such that $(U \times \operatorname{cl}(V)) \cap G(f)=\varnothing$ [2]. According to Theorem 7 of [1], each function $f: X \rightarrow Y$ of a topological space $X$ into a minimal Hausdorff space $Y$ with strongly-closed graph is continuous. (Note that Example 3 of [1] shows that the strongly-closed graph condition in Theorem 7 of [1] cannot be relaxed to a closed graph condition.)

2. Main result. Denote by $\delta$ the class of spaces containing the class of Hausdorff completely normal and fully normal spaces [3].

THEOREM. A Hausdorff space $Y$ is minimal Hausdorff if and only if for every topological space $X$ belonging to $\delta$, each function $f: X \rightarrow Y$ with a strongly-closed graph is continuous.

Proof. In view of Theorem 7 of [1], only the sufficiency requires proof. Assume that $Y$ is not minimal Hausdorff. By Theorem 5 of [1] there exists a net $f: \mathscr{D} \rightarrow Y$ with a unique $r$-accumulation point $q \in Y$ such that $f$ does not converge to $q$. Let $\infty \notin \mathscr{D}$ and define $X=\mathscr{D} \cup\{\infty\}$. Then the power set of $\mathscr{D}$ together with $\left\{T_{d} \cup\{\infty\} \mid d \in \mathcal{D}\right\}$ is a base for a topology $\sigma$ on $X$ making $(X, \sigma)$ a fully normal, completely normal Hausdorff space [2], [3]. Define $g: X \rightarrow Y$ by $g \mid \infty=g$ and $g(\infty)=q$. Using the fact that $q$ is the unique $r$ accumulation point of the net $f$, it follows that $G(g)$ is strongly-closed. The

Received by the editors October 13, 1975.

AMS (MOS) subject classifications (1970). Primary 54D20.

Key words and phrases. Minimal Hausdorff spaces, functions with strongly-closed graphs.

1 The concepts of $r$-convergence and $r$-accumulation point were first introduced by $N$. V. Velicko under the names of $\theta$-convergence and $\theta$-contact point, respectively, in $\boldsymbol{H}$-closed topological spaces, Mat. Sb. 70 (112) (1966), 98-112. 
identity function 1: $\mathscr{D} \rightarrow \mathscr{D}$ defines a net that converges to $\infty$. However, since $f$ does not converge to $q$, there exists an open set $V \subset Y$ containing $q$ with the property that $g\left(T_{d}\right) \cap(Y-V) \neq \varnothing$ for each $d \in \mathscr{D}$. Consequently, $g$ is not continuous at $x=\infty$. This contradiction establishes the proof.

\section{REFERENCES}

1. Larry L. Herrington and Paul E. Long, Characterizations of C-compact spaces, Proc. Amer. Math. Soc. 52 (1975), 417-426.

2. Characterizations of H-closed spaces, Proc. Amer. Math. Soc. 48 (1975), 469-475.

3. Shouro Kasahara, Characterizations of compactness and countable compactness, Proc. Japan Acad. 49 (1973), 523-524. MR 48 \# 7207.

Department of Mathematics and Physics, University of Arkansas at Pine Bluff, Pine BLUfF, ARKANSAS 71601

Department of Mathematics and Statistics, University of Arkansas at Fayetteville, FAYETTEVILLE, ARKANSAS 72701 